\title{
Spontaneous resolution of subfoveal perfluorocarbon
}

This article was published in the following Dove Press journal:

Clinical Ophthalmology

19 March 2015

Number of times this article has been viewed

\author{
Patrick Oellers' \\ Leon D Charkoudian² \\ Paul Hahn' \\ 'Department of Ophthalmology, \\ Duke University Medical Center, \\ Durham, NC, USA, ${ }^{2}$ Cape Fear Retinal \\ Associates, Wilmington, NC, USA
}

Correspondence: Paul Hahn

Department of Ophthalmology, Duke

University Medical Center, $235 \mathrm{I}$

Erwin Road, Box 3802, Durham, NC

27710, USA

$\mathrm{Tel}+|9| 9684563 \mid$

Fax +19196816474

Email paul.s.hahn@duke.edu

\begin{abstract}
Perfluorocarbon liquids (PFCL) have transformed the surgical approach to complex retinal detachments, but their use can be complicated by subretinal retention. Migration of peripherally located subretinal PFCL towards the fovea is well established, but spontaneous resolution of subfoveal PFCL without surgical evacuation or displacement has rarely been described. We present a case of a large single retained subfoveal PFCL droplet following vitrectomy with membrane peeling, inferior relaxing retinectomy, and silicone oil tamponade that demonstrated spontaneous resolution 3 weeks postoperatively, with subsequent anatomic and visual improvement. No residual subretinal PFCL was noted, and we hypothesize that the PFCL droplet spontaneously extruded through a transient hole created in the thinned retina overlying the droplet, which subsequently closed spontaneously. Further understanding of the mechanisms of PFCL migration and resolution may facilitate improved treatments for this complication.
\end{abstract}

Keywords: perfluorocarbon, subretinal, retained, resolution

\section{Introduction}

The unique chemical and physical properties of perfluorocarbon liquids (PFCL) have enabled internal displacement of subretinal fluid and stabilization of mobile retina, transforming the surgical approach to complex retinal detachments. Despite these advantages, use of PFCL may be complicated by inadvertent retention of PFCL droplets in the subretinal space. ${ }^{1}$ Subfoveal PFCL may be visually significant, and prompt surgical removal or displacement is indicated according to the visual prognosis. ${ }^{2}$ While peripheral subretinal PFCL can often be observed, migration of peripherally located droplets towards the fovea is well recognized, although the mechanisms of migration remain unknown. ${ }^{3}$ Spontaneous resolution of subfoveal PFCL without surgical evacuation or displacement has rarely been described. ${ }^{4}$ We present a case of a large single retained subfoveal PFCL droplet that demonstrated spontaneous resolution 3 weeks postoperatively.

\section{Case report}

A 72-year-old man was referred with a history of recurrent retinal detachment in the right eye (Figure 1A) repaired with prior vitrectomy and subsequent encircling scleral buckle, vitrectomy, and silicone oil. Upon referral, he was noted to have counting fingers visual acuity with a recurrent macula-involving retinal detachment under silicone oil with diffuse posterior and anterior preretinal grade $\mathrm{C}$ proliferative vitreoretinopathy. Retinal detachment repair was subsequently performed with 23-gauge vitrectomy, extensive peeling of preretinal posterior and anterior membranes, stabilization of the posterior pole with PFCL (Perfluoron ${ }^{\circledR}$, Alcon, Fort Worth, TX, USA), $180^{\circ}$ inferior relaxing retinectomy, laser photocoagulation, and 1,000 centistoke silicone oil 

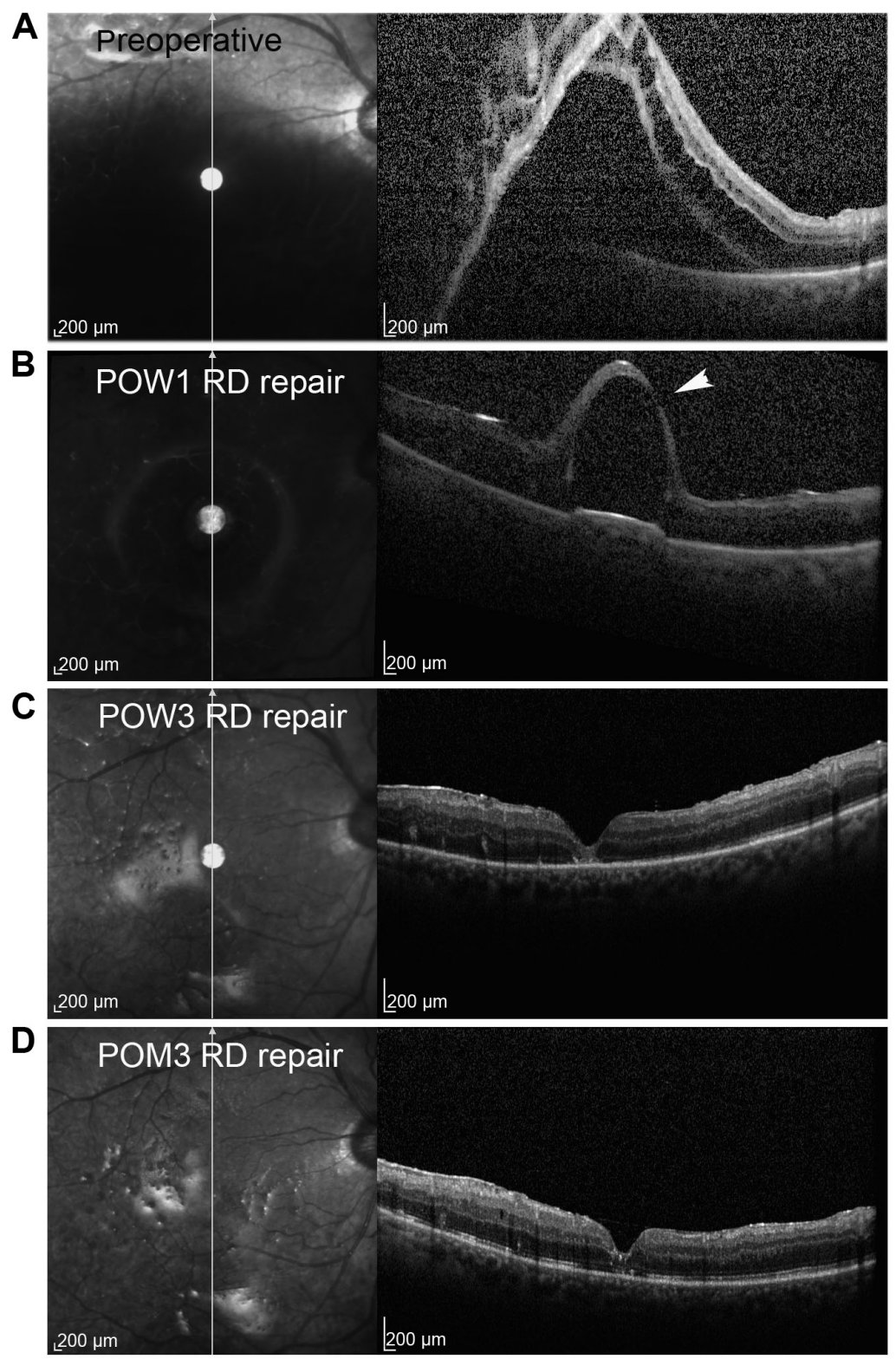

Figure I Spectral domain optical coherence tomographic images with corresponding scanning laser ophthalmoscopic images of the left macula.

Notes: (A) Preoperatively, the retina is detached under silicone oil. (B) At I week following recurrent retinal detachment repair, the retina remains attached with a large subfoveal PFCL droplet. There is a focal area of severe thinning with a possible retinal defect (arrowhead) overlying the droplet. (C) At 3 weeks following repair, without any surgical intervention, the retina remains attached, but the PFCL droplet is no longer identified and is replaced by granular changes at the outer retina with a restored foveal contour. (D) By 3 months following repair, these granular changes have improved, with visualization of outer retinal structures.

Abbreviations: PFCL, perfluorocarbon liquid; RD, retinal detachment; POW, postoperative week; POM, postoperative month.

tamponade. On postoperative day 1 and then at postoperative week 1 , the patient had counting fingers vision with retinal attachment under silicone oil, but a large subfoveal PFCL droplet was noted on ophthalmoscopy and confirmed with optical coherence tomography (OCT) imaging, which revealed a focal area of severely thinned retinal tissue overlying the droplet (Figure 1B). The decision was made with the patient to proceed with surgical displacement of the subfoveal PFCL droplet, but for personal reasons, he required delaying surgery until the third postoperative week. On postoperative day 21 , in the preoperative holding area, he casually noted sudden improvement in his vision 2 days prior. Ophthalmoscopy was performed, demonstrating absence of previously seen subretinal PFCL at the fovea, at the retinectomy edge, or anywhere in the subretinal space. OCT performed that day revealed a restored foveal depression with focal outer retinal granulation and thinning (Figure 1C). Without any surgical intervention, these architectural changes improved over the subsequent months, with restoration of the ellipsoid zone band at the fovea (Figure 1D). His visual acuity remained 
at the counting fingers level until removal of his silicone oil 4 months following placement. Subretinal or intravitreal PFCL was never identified, including intraoperatively during oil removal. His visual acuity improved to $20 / 150$ at one day following oil removal and to 20/70 at 6 weeks following oil removal, with sustained retinal attachment, absence of any retained PFCL, and continued improvement of the retinal contour and microarchitecture.

\section{Discussion}

Subretinal PFCL retention is a well described complication following its use in retinal detachment repair. ${ }^{1}$ While migration of subretinal PFCL toward the fovea is a recognized phenomenon, ${ }^{3}$ spontaneous resolution of subfoveal PFCL has rarely been described. In one recent report, a cluster of subfoveal PFCL droplets spontaneously migrated to the inferior subretinal periphery after being stable for several years following retinal detachment repair. ${ }^{4}$ To the authors' knowledge, no other reports of spontaneous subfoveal PFCL resolution have been documented.

In this report, we demonstrate a second case of spontaneous resolution of subfoveal PFCL. Surprisingly, careful inspection of the posterior and peripheral retina did not reveal any subretinal PFCL droplets following resolution, including at the retinectomy edge. It is possible that the single large PFCL droplet separated into smaller subretinal droplets that were not ophthalmoscopically visible, but we feel this is unlikely, as even small PFCL droplets in the subretinal space are usually seen. It may be possible that the large PFCL droplet was hidden by other structures, but we feel this is also unlikely given the successful reattachment without recurrent proliferative vitreoretinopathy and a clear view under silicone oil. Alternatively, PFCL droplets may have migrated through the subretinal space past the retinectomy edge into the vitreous cavity, but we think this is also unlikely given the sustained retinal attachment including at the lasered retinectomy edge. Rather, we hypothesize, based on a putative defect identified on OCT within the thin retina overlying the PFCL droplet (Figure 1B, arrowhead) that the surface tension of the PFCL droplet resulted in tangential forces that, compounded by accompanying peeling of preretinal proliferative vitreoretinopathy membranes, resulted in a focal overlying defect at the fovea. We suggest that the PFCL droplet extruded through this retinal defect into the vitreous cavity, where it remained undetected, but was subsequently removed during silicone oil removal. The possibility of retinal hole formation due to chronic subretinal PFCL retention with a very thin overlying retina has been entertained in the literature. ${ }^{5}$ In our case, we hypothesize that resolution of the tangential forces after extrusion of the PFCL droplet led to spontaneous closure of the hole, resulting in a course of granulation and closure similar to that seen following full-thickness macular hole repair.

This case demonstrates that resolution of subfoveal PFCL can occur spontaneously either through presumed extrusion into the vitreous cavity or, as has been previously reported, through peripheral migration in the subretinal space. The mechanism and triggering forces of these rare events are poorly understood, and we advocate prompt surgical removal or displacement of subfoveal PFCL. In the future, improved understanding of the mechanisms of spontaneous resolution may enable the development of nonsurgical methods to displace subfoveal PFCL.

\section{Disclosure}

$\mathrm{PH}$ has a consulting agreement unrelated to the submitted work with Second Sight Medical Products, Inc. The authors have no other conflicts of interest in this work.

\section{References}

1. Garcia-Valenzuela E, Ito Y, Abrams GW. Risk factors for retention of subretinal perfluorocarbon liquid in vitreoretinal surgery. Retina. 2004; 24(5):746-752.

2. Shulman M, Sepah YJ, Chang S, Abrams GW, Do DV, Nguyen QD. Management of retained subretinal perfluorocarbon liquid. Ophthalmic Surg Lasers Imaging Retina. 2013;44(6):577-583.

3. Tewari A, Eliott D, Singh CN, Garcia-Valenzuela E, Ito Y, Abrams GW. Changes in retinal sensitivity from retained subretinal perfluorocarbon liquid. Retina. 2009;29(2):248-250.

4. Yiu G, Fekrat S, Hahn P. Spontaneous peripheral migration of subfoveal perfluorocarbon. Retina. 2014;34(11):2315-2316.

5. Cohen SY, Dubois L, Elmaleh C. Retinal hole as a complication of long-standing subretinal perfluorocarbon liquid. Retina. 2006;26(7): 843-844.
Clinical Ophthalmology

\section{Publish your work in this journal}

Clinical Ophthalmology is an international, peer-reviewed journal covering all subspecialties within ophthalmology. Key topics include: Optometry; Visual science; Pharmacology and drug therapy in eye diseases; Basic Sciences; Primary and Secondary eye care; Patient Safety and Quality of Care Improvements. This journal is indexed on
Dovepress

PubMed Central and CAS, and is the official journal of The Society of Clinical Ophthalmology (SCO). The manuscript management system is completely online and includes a very quick and fair peer-review system, which is all easy to use. Visit http://www.dovepress.com/ testimonials.php to read real quotes from published authors. 